\title{
Spectra of Poynting-flux powered GRB outflows
}

\author{
D. Giannios ${ }^{1,2,3}$ and H. C. Spruit ${ }^{1}$ \\ 1 Max Planck Institute for Astrophysics, Box 1317, 85741 Garching, Germany \\ e-mail: giannios@mpa-garching.mpg.de \\ ${ }^{2}$ University of Crete, Physics Department, PO Box 2208, 71003 Heraklion, Crete, Greece \\ ${ }^{3}$ Foundation for Research and Technology-Hellas, 71110 Heraklion, Crete, Greece
}

Received 8 January 2004 / Accepted 25 August 2004

\begin{abstract}
We investigate the production of the gamma-ray spectrum of a Poynting-flux dominated GRB outflow. The very high magnetic field strengths (super-equipartition) in such a flow lead to very efficient synchrotron emission. In contrast with internal shocks, dissipation of magnetic energy by reconnection is gradual and does not produce the spectrum of cooling electrons associated with shock acceleration. We show that a spectrum with a break in the BATSE energy range is produced, instead, if the magnetic dissipation heats a small $\left(\sim 10^{-4}\right)$ population of electrons.
\end{abstract}

Key words. gamma rays: bursts - magnetohydrodynamics (MHD) - radiation mechanisms: non-thermal

\section{Introduction}

Magnetic fields are an attractive ingredient in central engine models of $\gamma$-ray bursts because of their ability to transfer large amounts of energy across (near) vacuum and depositing it into a small amount of matter. The energy flux in such magnetically powered, or "Poynting-flux dominated" outflows (Thompson 1994; Lyutikov \& Blandford 2003; Drenkhahn 2002) is initially in electromagnetic form. To produce a GRB, this energy flux has to be transferred to the matter in such a way as to produce both bulk flow speed and the right amount of nonthermal radiation.

Poynting-flux dominated outflows come in 2 basic types: the axisymmetric or "DC" models and the nonaxisymmetric or "AC" ones (for discussions of these models see Lyutikov \& Blandford 2003; Drenkhahn 2002 (Paper I); Spruit \& Drenkhahn 2002 (Paper II)). An axisymmetric, rotating magnetic field can accelerate flows by the magnetocentrifugal mechanism. Instabilities in the outflow can dissipate some of the magnetic energy to produce heating, acceleration of fast particles and ultimately nonthermal radiation. In the AC model on the other hand, the rotating magnetic field of the central engine is nonaxisymmetric, producing an outflow in which the field lines change directions rapidly, at the rotation frequency of the central engine or higher. Dissipation of magnetic energy by reconnection in this flow is much faster than in the DC model, and the acceleration of the outflow very efficient, though by a somewhat different mechanism than in the magnetocentrifugal process (Papers I; II, for a summary see Spruit \& Drenkhahn 2003).

In both these models, the observed nonthermal radiation is produced by dissipation of magnetic energy by reconnection.
Details of the reconnection process matter for the radiation produced, but are rather poorly understood at present. Significant parts of the relevant plasma physics are not known, especially under the relativistic conditions of a GRB. This hampers for the moment the development of models for the prompt radiation to be expected from a magnetically powered GRB outflow. Nevertheless, a few reasonably established facts already help in limiting the possibilities.

In this paper we develop the consequences of assuming magnetic reconnection as the source of gamma-radiation from a GRB. Like the internal shock model, the model has a free parameter representing the fraction of the electrons that takes up the dissipated energy and produces the synchrotron radiation. It has one degree of freedom less, however, since the magnetic field strength in the flow follows within narrow limits from the energetics, once the choice for an AC or DC model is made. In the developments below, an $\mathrm{AC}$ model is assumed for quantitative results.

An important diagnostic of the prompt emission is the break in the Gamma-ray spectrum. This break is usually assumed to represent the synchrotron spectrum from a distribution of electrons with a lower energy cutoff, as is produced by the synchrotron cooling of quasi-instantaneously accelerated electrons. This may be appropriate for an internal shock model. The idea has also been carried over to magnetic prompt emission models by Sikora et al. (2003). It turns out, however, that magnetic reconnection is not nearly instantaneous enough to produce such a distribution. In magnetic reconnection, the electron distribution will reflect, instead, the local instantaneous balance between synchrotron cooling and heating by the reconnection-related electric fields. 


\section{Magnetically powered outflows}

The outflow carries free energy in the form of wound-up magnetic field lines, which can be extracted through reconnection processes. For reconnection processes to take place, differently oriented field lines must come close to each other. The length scale on which the orientation of magnetic field lines changes controls the speed of field dissipation; the smaller the length scale the faster the field decay. In DC models, the field lines are in the same direction, and dissipation has to wait until global instabilities have caused changes in direction between nearby field lines. As a result the dissipation in such models tends to happen at a rather large distance, of order $10^{17} \mathrm{~cm}$ (Lyutikov \& Blandford 2003).

We consider here the outflow from a nonaxisymmetric rotator. This configuration gives rise to a "striped" wind (Coroniti 1990; see also Spruit et al. 2001), where the magnetic field varies with a wavelength of $\lambda \sim 2 \pi c / \Omega$ (in the source's frame). A detailed description of the model has been given previously (Papers I and II). In this section we summarize the main ingredients of the model and its assumptions.

\subsection{Reconnection in a relativistic magnetic field}

Both the dynamics of the flow and the radiative energy release are governed by the rate of magnetic reconnection in the flow. The magnetic energy flux is of the same order as the kinetic energy flux $\Gamma \dot{m} c^{2}$ where $\dot{m}=\dot{M} / 4 \pi$ is the (baryonic) mass flux per steradian and $\Gamma$ the bulk Lorentz factor. In a frame comoving with the flow, the magnetic energy density is then of the same order as the rest mass energy density, i.e. the field is relativistic (Alfvén speed of the order of the speed of light) and highly super-equipartition.

The outflow speed of the plasma from a reconnection region is of the order of the Alfvén speed, rather independent of the particular reconnection model. Under relativistic conditions (energy density in the magnetic field larger than the rest mass energy density), Blackman \& Field (1994a) (see also Blackman $\&$ Field 1994b) find that the inflow speed into the reconnection layer is increased by the relativistic kinematics, and is also subrelativistic. This has also been found by Lyutikov \& Uzdensky (2003). Thus we may assume that the velocity $v_{\text {rec }}$ at which the magnetic energy density $B^{2} / 8 \pi$ flows across a reconnection boundary is of the order of the speed of light

$v_{\text {rec }}=\epsilon v_{\mathrm{A}}$,

where $v_{\mathrm{A}} \approx c$ and $\epsilon$ is of order unity. We keep this parameter in the calculations, but consider it a relatively well determined quantity, conservatively set at $\epsilon=0.1$ for quantitative estimates.

\subsection{The dynamics of the flow}

Near the source the flow is accelerated magnetocentrifugally up to a distance around the Alfvén radius. Then it becomes asymptotically radial. The poloidal and azimuthal components are similar in magnitude; however, from that point on their magnitude scales as $B_{\phi} / B_{r} \sim r$, resulting in a negligible radial component at larger distances. In Poynting-flux dominated outflows the Alfvén radius is almost equal to the light radius $c / \Omega$. So for distances $r>c / \Omega$ the flow can be assumed to be purely radial with a dominant azimuthal component of the magnetic field.

From this point on we focus on the dynamics of the flow, as well as on its radiative properties at distances $r>r_{0} \sim$ a few $c / \Omega$. An important parameter of the model is the ratio $\sigma_{0}$ of the Poynting flux to the kinetic flux at the initial radius

$\sigma_{0}=\frac{L_{\mathrm{B}, 0}}{L_{\mathrm{kin}, 0}}=\frac{\beta_{0}\left(B_{0} r_{0}\right)^{2}}{4 \pi \Gamma_{0} \dot{M} c}$

where $\beta=v / c, B_{0}$ and $\Gamma_{0}$ are the magnetic field strength and the bulk Lorentz factor at the radius $r_{0}$ and $\dot{M}$ is the mass flux per sterad. The flow starts at $r_{0}$ with the Alfvén speed $\left(\Gamma_{0}=\right.$ $\left.\sqrt{\sigma_{0}+1}\right)$. In the GRB case the flow must start highly Poyntingflux dominated with $\sigma_{0} \gtrsim 100$ so that $\Gamma \gg 1$ at all distances. So we can safely set $\sigma_{0}+1 \simeq \sigma_{0}$ and $\beta \simeq 1$.

There is also a simple relation that links the total luminosity of the flow to the mass flux

$L=\sigma_{0} \Gamma_{0} \dot{M} c^{2}$.

A characteristic value of the asymptotic Lorentz factor of the flow is

$\Gamma_{\infty} \approx \sigma_{0} \Gamma_{0}$

Its actual value will differ, depending on which fraction of the Poynting flux is converted to kinetic energy. In the AC model, this is about $50 \%$ if most of the dissipation takes place outside the photosphere, and larger (up to $100 \%$ ) when dissipation is near or inside the photosphere.

We will apply the results to cases where magnetic dissipation is well outside the photosphere so that the asymptotic kinetic luminosity is similar to the initial Poynting flux. In the region $r<r_{\mathrm{s}}$ where the Poynting flux has not yet dissipated significantly, this implies the approximate relation

$\frac{B^{2}}{8 \pi} c \approx \Gamma_{\infty} \rho c^{3}$,

in the frame of the central engine, or

$\frac{B^{\prime 2}}{8 \pi} \approx \frac{\Gamma_{\infty}}{\Gamma} \rho^{\prime} c^{2}, \quad\left(r \lesssim r_{\mathrm{s}}\right)$

in a frame comoving with the bulk flow (indicated by primes). Thus, as mentioned above, the magnetic field in the outflow is relativistic, up to the saturation radius $r_{\mathrm{s}}$ where most of the dissipation takes place.

With magnetic dissipation scaling with the Alfvén speed as discussed above, the acceleration of the flow can be computed in detail, including the gas and radiation pressures where relevant. The results of detailed numerical calculations where reported in Paper II. The acceleration of the flow stops when most of the free magnetic energy has been dissipated; this happens at a distance $r_{\mathrm{s}}$. In the intermediate range of radii $r_{0} \ll r \ll r_{\mathrm{s}}$ the equations simplify and an analytic solution is possible (Paper I). The analytic solution has been found to be fairly close 
to the numerical results and will be used in this work so as to make predictions on the radiative properties of the flow.

In the intermediate range of radii the bulk Lorentz factor of the flow scales as $\Gamma \propto r^{1 / 3}$, while beyond the saturation radius it can be approximated as constant

$\Gamma=\Gamma_{\infty}\left(r / r_{\mathrm{s}}\right)^{1 / 3}$ for $r<r_{\mathrm{s}}$,

$\Gamma=\Gamma_{\infty}=\sigma_{0}^{3 / 2}$ for $r>r_{\mathrm{s}}$,

where

$r_{\mathrm{s}}=\pi c \Gamma_{\infty}^{2} /(3 \Omega \epsilon)$,

or, in terms of the light cylinder radius $r_{1}$,

$r_{\mathrm{s}} / r_{1}=\frac{\pi}{2 \epsilon} \Gamma_{\infty}^{2}$.

For canonical GRB parameters $\left(\Gamma=300, \epsilon=0.1, r_{1}=10^{7} \mathrm{~cm}\right.$, it is at $r_{\mathrm{s}} \sim 10^{13} \mathrm{~cm}$, a factor 10-100 outside the Thomson photosphere.

We can see how much magnetic energy is dissipated in the flow (in the central engine's frame) as a function of radius following the Poynting flux in the flow. The latter is given by the expression (Paper I)

$L_{\mathrm{B}}=\beta c \frac{(r B)^{2}}{4 \pi}=L\left(1-\frac{\Gamma}{\sigma_{0}^{3 / 2}}\right)$,

where $L, L_{\mathrm{B}}$ are the the total flux and the Poynting flux of the flow per steradian. From Eqs. (11) and (7) one can see that the Poynting flux in the flow drops as a function of distance. This reduction corresponds to the magnetic energy that is gradually dissipated in the flow. The energy rate $\mathrm{d} \dot{E}_{\text {rel }}$ that is released in a shell $(r, r+\mathrm{d} r)$ of the flow will be

$\mathrm{d} \dot{E}_{\text {rel }}=-\frac{\mathrm{d} L_{\mathrm{B}}}{\mathrm{d} r} \cdot \mathrm{d} r=\frac{0.049 L}{r_{11}^{2 / 3}} \frac{(\epsilon \Omega)_{3}^{1 / 3}}{\sigma_{0,2}} \mathrm{~d} r_{11}$,

where $r=10^{11} r_{11} \mathrm{~cm}$. So in the frame of the central engine $\mathrm{d} \dot{E}_{\text {rel }} \propto \mathrm{d} r / r^{2 / 3}$. After integration in a range of radii we find $\dot{E} \propto r^{1 / 3}$. So, most of the energy is actually dissipated in the outer regions of the flow, close to the saturation radius.

Other physical quantities of the flow that will be important are the proper density $\rho$, the magnetic field strength $B$, and the location of the Thomson photosphere $r_{\mathrm{ph}}$. Combining the continuity equation $\dot{M}=r^{2} \Gamma \rho c$ with Eqs. (3) and (7) we have for the proper density

$\rho=\frac{\dot{M}}{r^{2} \Gamma c}=\frac{L}{\sigma_{0,2}^{3 / 2} r^{2} \Gamma c^{3}}=\frac{2.5 \times 10^{-7}}{r_{11}^{7 / 3}} \frac{L_{52}}{\sigma_{0,2}^{2}(\epsilon \Omega)^{1 / 3}} \mathrm{~g} / \mathrm{cm}^{3}$,

where $\dot{M}$ is the mass flux per steradian and $L=$ $10^{52} L_{52} \mathrm{erg} / \mathrm{s} / \mathrm{sterad}$ is a typical value for the observed GRB luminosities taking into account the strong evidence for highly collimated outflows (Frail et al. 2001).

Using Eqs. (11) and (7) (for $\Gamma \ll \Gamma_{\infty}$ ) the comoving magnetic field strength will be

$B^{\prime}=B / \Gamma=\frac{25 \times 10^{7}}{r_{11}^{4 / 3}} \frac{L_{52}^{1 / 2}}{(\epsilon \Omega)_{3}^{1 / 3} \sigma_{0,2}^{1 / 2}} \quad$ Gauss.
The radius of the photosphere of the flow depends on the baryon loading and the energy flux. For large bulk Lorentz factors it is given by integrating the expression (cf. Abramowicz et al. 1991) $\mathrm{d} \tau=\Gamma(1-\beta) k_{\mathrm{es}} \rho \mathrm{d} r$ from $r$ to $\infty$ to find

$\tau=\frac{20}{r_{11}^{5 / 3}} \frac{L_{52}}{\sigma_{0,2}^{5 / 2}(\epsilon \Omega)_{3}^{2 / 3}}$.

Setting $\tau\left(r_{\mathrm{ph}}\right)=1$ and solving for the location of the photosphere, we find its position as a function of the parameters of the flow

$r_{\mathrm{ph}, 11}=6 \frac{L_{52}^{3 / 5}}{\sigma_{0,2}^{3 / 2}(\epsilon \Omega)_{3}^{2 / 5}}$

\section{The reconnection layer}

\subsection{Time scales}

Let the reconnection layers have a lateral size $L$ (given by the wavelength of the field reversals) and an as yet unknown thickness $\delta$. Matter and magnetic field lines are advected to the reconnection region with the reconnection velocity $v_{\text {rec }}$ through the upper and lower surfaces of the layer. The material is pulled out of the reconnection region through the sides of the layer with speeds comparable to the Alfvén speed by magnetic tension forces. While $\delta$ depends strongly on the poorly known reconnection physics, the outflow speed is approximately known, since the Alfvén speed is of the order of the speed of light, as noted above. The width $L$ of the reconnection layer is given by the intrinsic length scale in the flow, namely the wavelength of field reversals. Hence, in a frame comoving with the bulk flow $L \approx \Gamma \pi c / \Omega$. The residence time of matter in the reconnection layer is therefore of the order

$\tau_{\mathrm{r}}=L / v_{\mathrm{A}} \approx \Gamma \pi / \Omega=\pi r_{\mathrm{l}} / c$

where $r_{1}$ is the light cylinder radius of the central engine.

This time scale can be compared with the cooling time of the electrons in the reconnection layer. On account of the very high field strengths, the dominant radiation process is most likely synchrotron emission. Because the details of the particle acceleration processes in the reconnection layer are unknown, we assume that the energy dissipated goes mostly into a fraction $\xi \ll 1$ of the electrons, which as a result attain a typical Lorentz factor $\gamma$. The parameter $\xi$ plays the same role as its counterpart in the internal shock model. Note, however, that there is no additional parameter for the strength of the magnetic field, since this is fixed by the Poynting flux model.

If the characteristic Lorentz factor of the radiating electrons is such that the radiation seen in the observer's frame has energy $h v_{\text {obs }} / m_{\mathrm{e}} c^{2}=x_{\mathrm{obs}} \approx 1$, the cooling time of the electrons (in a comoving frame) can be written as

$\tau_{\mathrm{s}}=6 \pi \sigma_{\mathrm{T}}^{-1} \Gamma^{1 / 2} x_{\mathrm{obs}}^{-1 / 2}(e \hbar / c)^{1 / 2} B^{\prime-3 / 2}$.

The electron cooling time is very short due to the high magnetic field strengths that make the electrons radiate efficiently. The electrons thus stay cold, and if most of the magnetic energy is dissipated to the electrons, the flow as a whole stays cold. 
The reconnection time scale $\tau_{\mathrm{r}}$ is of the order of the light travel time over the length scale of the magnetic field in the comoving frame

$\tau_{\mathrm{r}} \approx \pi r_{1} \Gamma / c$.

In terms of the energy flux in the burst, and making use of Eq. (8), the ratio of the two time scales becomes

$\tau_{\mathrm{s}} / \tau_{\mathrm{r}} \approx 10^{-7}\left(r_{1} / 10^{7}\right)^{1 / 2} x_{\mathrm{obs}}^{-1 / 2}\left(r / r_{\mathrm{s}}\right)^{11 / 6} L_{52}^{-3 / 4}(\epsilon / 0.1)^{-3 / 2}$.

The synchrotron cooling time is thus very short compared with the residence time of the radiating electrons in the acceleration region. This has the consequence that the usual approximation of sudden acceleration followed by cooling of the electron population made in the internal shock model is not a good model for the synchrotron emission from magnetic reconnection. Rather, the electron energy distribution must be close to an equilibrium between synchrotron cooling and heating through reconnection.

\subsection{Energetics of the reconnection region}

In this section we estimate the rate at which magnetic energy is released in a reconnection layer. Matter and magnetic field lines are advected through the upper and lower surfaces of the layer with the reconnection velocity $v_{\text {rec }}$. The flux of the incoming magnetic energy in the reconnection layer is

$F_{\mathrm{B}}=2 \frac{B^{\prime 2}}{8 \pi} v_{\mathrm{rec}}$,

where the factor 2 accounts for the two sides of the layer. This magnetic flux is dissipated over the thickness $\delta$ of the layer resulting in a rate of magnetic energy density released

$\dot{E}_{\mathrm{dis}}=\frac{B^{\prime 2}}{4 \pi} \frac{v_{\text {rec }}}{\delta}$

The material leaves the reconnection region through the side of the layer with speeds comparable to the Alfvén speed. If the proper density of the flow is $\rho_{\text {in }}$ and $\rho_{\text {out }}$ inside and outside the layer respectively, then mass conservation gives

$L \rho_{\text {out }} v_{\text {rec }} \Gamma_{\text {rec }}=\delta \rho_{\text {in }} v_{\mathrm{A}, \text { co }} \Gamma_{\mathrm{A}, \mathrm{co}}$,

where $v_{\mathrm{A}, \mathrm{co}}$ is the comoving Alfvén speed, with its corresponding Lorentz factor

$\Gamma_{\mathrm{A}, \mathrm{co}}=\sqrt{1+\sigma}, \quad \sigma=\frac{B_{\mathrm{co}}^{2}}{4 \pi \rho c^{2}}=\frac{6.8}{r_{11}^{1 / 3}} \frac{\sigma_{0,2}}{(\epsilon \Omega)_{3}^{1 / 3}}$.

For typical distances of interest $r_{11} \gtrsim 0.1, \Gamma_{\mathrm{A}, \mathrm{co}} \sim$ a few. Finally, $\Gamma_{\text {rec }}$ is the Lorentz factor that corresponds to the reconnection velocity. Since $v_{\text {rec }}=\epsilon v_{\mathrm{A}, \mathrm{co}}$, and the reconnection speed parameter $\epsilon$ is not close to unity, we have $\Gamma_{\text {rec }} \simeq 1$.

Mass conservation (Eq. (23)) provides a constraint on $\delta$, but something has to be known about the density ratio $\rho_{\text {out }} / \rho_{\text {in }}$ for this to be useful. This in turn depends on the efficiency with which the dissipated energy is radiated away. If the energy stays in the particles, then the particle pressure can compensate the decline of the magnetic energy pressure in the dissipation layer and the flow is essentially incompressible. If, however, the particles radiate very efficiently, they stay relatively cool, resulting in a highly compressible flow (i.e. $\rho_{\text {in }} \gg \rho_{\text {out }}$ ). Thus, the thickness of the reconnection layer remains somewhat uncertain, but it turns out that this has no effect on the spectral estimates made in the next section, since the value of $\rho_{\text {in }}$ drops out.

For parameters of the model relevant to GRBs, the protons are inefficient radiators owing to their high mass in contrast to electrons. It is often argued that in reconnection most of the magnetic energy is dissipated into the electrons rather than the ions, on account of their greater mobility in the electric fields generated in the reconnection region. It is not known to what extent this still holds for the relativistic reconnection environment of a GRB. For completeness we keep the fraction dissipated into the electrons, $f_{\mathrm{e}}$ as a free parameter. If $f_{\mathrm{e}} \ll 1$, most of the energy is transfered to the protons and, unless there is a very efficient mechanism of energy coupling between electrons and protons, most of the energy will serve to accelerate the bulk flow through adiabatic cooling of the protons. In this case the radiative efficiency of the flow becomes too low to explain a GRB. As in the internal shock model, we assume that this is not the case, i.e. $f_{\mathrm{e}}$ is taken of order unity.

Another parameter of the model is the fraction $\xi$ of the electrons that will be accelerated in the reconnection region. The two parameters $f_{\mathrm{e}}$ and $\xi$ correspond to the same degrees of freedom as in the internal shock model, where a large fraction of the energy that is dissipated in the shocks accelerates the electrons, and another fraction is assumed to build random magnetic fields.

We can estimate the characteristic Lorentz factor to which the electrons are accelerated in the reconnection layers at different distances from the central engine. The electrons are heated at a rate

$\dot{E}_{\text {heat }}=f_{\mathrm{e}} \dot{E}_{\text {dis }}$.

An electron moving with a Lorentz factor $\gamma_{\mathrm{ch}}$ in a magnetic field of strength $B^{\prime}$ will radiate at a rate $\sigma_{T} v^{2} \gamma_{\mathrm{ch}}^{2} B^{\prime 2} /(6 \pi c)$. If $\gamma_{\mathrm{ch}}$ is the characteristic Lorentz factor of the electron distribution then the cooling rate per unit volume due to synchrotron loss in the optical thin regime will be

$\dot{E}_{\mathrm{cool}}=\frac{\xi \rho_{\mathrm{in}}}{m_{\mathrm{p}}} \frac{\sigma_{\mathrm{T}} v^{2} \gamma_{\mathrm{ch}}^{2} B^{\prime 2}}{6 \pi c}$.

Inside the reconnection layer electrons are accelerated until $\dot{E}_{\text {heat }}=\dot{E}_{\text {cool }}$. Using Eqs. (25), (26) and (22) we find

$\beta_{\mathrm{ch}} \gamma_{\mathrm{ch}}=7 \cdot r_{11} \frac{\sigma_{0,2}^{3 / 4} \Omega_{4}^{1 / 2}}{\xi_{-4}^{1 / 2} L_{52}^{1 / 2}}$

where we have also set $f_{\mathrm{e}} \Gamma_{\mathrm{A}, \mathrm{co}}=1$ (i.e. $f_{\mathrm{e}} \simeq 0.5$ ) and $\rho_{\text {out }}=\rho$ (Eq. (13)). We have also normalized $\xi=10^{-4} \xi_{-4}$.

Equation (27) shows that the characteristic energy of the electrons actually turns out to be independent of the uncertain thickness $\delta$ of the reconnection region (related to the effective compressibility of the reconnection flow). This can be understood by the following consideration: high compressibility means small thickness $\delta$ of the reconnection layer and more 
energy dissipation per unit volume in the reconnection sites. On the other hand, this energy has to be shared among more particles (since the density is higher), resulting in the same heating rate per particle.

\section{The spectrum}

In this section we consider the radiation from reconnection regions in the outflow, under the assumptions that i) the dissipated energy goes into a fraction $\xi$ of the electrons; and ii) the electrons thus heated have a narrow or quasi-thermal distribution. The spectrum of the burst is then the synchrotron spectrum of this distribution, folded over the distance from the source and Doppler shifted to the observer using the radial dependences given in Sect. 2.2.

\subsection{The peak frequency of the spectrum}

Equation (27) is simplified in the ultrarelativistic regime, where $\gamma_{\mathrm{ch}} \propto r$. The characteristic photon energy at which the electrons emit (in the comoving frame) scales as $v^{\prime} \propto \gamma_{\mathrm{ch}}^{2} B^{\prime} \propto r^{2 / 3}$ and is Doppler boosted in the central engine's frame to an energy $v_{\mathrm{ch}} \propto \Gamma v^{\prime} \propto r$. So the most energetic photons are expected to be emitted in the outer parts of the flow close to the saturation radius. Since this is also where most of the magnetic energy is dissipated, these photons will give rise to the peak of the $v \cdot f_{v}$ spectrum.

More quantitatively, in the central engine's frame the characteristic frequency emitted as a function of radius is $v_{\mathrm{ch}}=$ $3 / 2 \Gamma \gamma_{\mathrm{ch}}^{2} v_{\mathrm{L}}$, where $v_{\mathrm{L}}=e B^{\prime} /(2 \pi m c)$ is the Larmor frequency. Using Eqs. (14), (7), and (27) we get in the relativistic limit

$X_{\mathrm{ch}}=\frac{h v_{\mathrm{ch}}}{m c^{2}} \approx 6 \times 10^{-3} r_{11} \frac{\Omega_{4}\left(\Gamma_{\infty} / 300\right)}{\xi_{-4} L_{52}^{1 / 2}}$.

$X_{\mathrm{ch}}$ becomes maximum at the saturation radius $r_{\mathrm{s}}$. Combining Eqs. (28) and (9):

$X_{\mathrm{ch}, \max }=0.3 \frac{\left(\Gamma_{\infty} / 300\right)^{3}}{\xi_{-4} \epsilon_{-1} L_{52}^{1 / 2}}$.

The peak in the $v \cdot f_{v}$ spectrum, which corresponds to the break of the Band spectrum (Band et al. 1993), is in the range $X_{\mathrm{br}} \sim(0.1-1)$. In the central engine's frame (assuming a typical redshift $\sim 1)$ the same peak is in the range $\sim(0.2-2)$. In the case that all the electrons are accelerated in the reconnection region $(\xi=1)$, if the peak is to be in the observed range, we must either have $\epsilon \ll 0.1$, or $\Gamma_{\infty} \gtrsim 1000$.

If, however, only a small fraction $\left(\xi \sim 10^{-4}\right)$ of the electrons is accelerated, values $\epsilon \simeq 0.1$ and $\Gamma_{\infty} \simeq 300$ are compatible with the characteristic of the prompt radiation. When specific values of the parameters have to be chosen for arithmetic examples, these values will be used in what follows.

\subsection{Spectral energy distribution}

For a prediction of the spectrum the actual electron distribution is needed, not only a characteristic Lorentz factor. The electron distribution depends on the acceleration mechanisms that

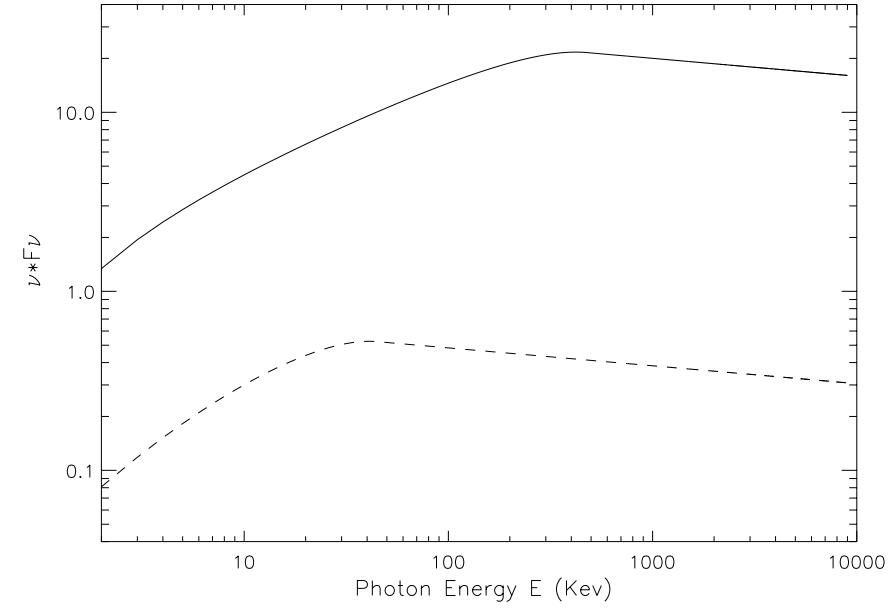

Fig. 1. Illustrative example of the spectrum (in the source's frame) emitted by a power law $\gamma^{-p}$ distribution of electrons in the reconnection centers. In this example $p=3.2$ resulting in the high energy slope of the spectrum. The other parameters are $\epsilon=0.1, \Omega=10^{4} \mathrm{rad} / \mathrm{s}$, $\sigma_{0}=100, L=3.2 \times 10^{51} \mathrm{erg} / \mathrm{s} / \mathrm{steradian}$ and $\xi=2 \times 10^{-3}$. The dashed line corresponds to $L=10^{50} \mathrm{erg} / \mathrm{s} /$ steradian and $\sigma_{0}=40$, parameters typical for X-ray flashes.

take place in the reconnection layers and on the cooling of the electrons. A thermal distribution is expected when particles can exchange energy efficiently. For the conditions expected in the reconnection layer, the heating and synchrotron cooling time scales can be shown to be orders of magnitude shorter than the energy exchange time scale due to Coulomb collisions. So Coulomb collisions are inefficient at thermalizing the electrons in the reconnection sites.

Another thermalization mechanism is synchrotron absorption. When the bulk of the radiating energy of the particles is reabsorbed, this provides an efficient channel of energy exchange between the particles, and a thermal distribution is expected. The role of the synchrotron absorption will be explored in the next section, where it will be shown that at distances comparable to or larger than the Thomson photosphere, electrons emit mostly in an environment optically thin to synchrotron radiation.

Under these conditions, at radii larger than the photospheric radius non-thermal electron distributions may appear. As an illustrative example we assume that the accelerated electrons have a power law distribution $N(\gamma) \propto \gamma^{-p}$, with a lower cutoff at the characteristic Lorentz factor as defined in Eq. (29) and $p>2$. The spectrum is obtained by integration over this distribution and over all contributing radii (those outside the photosphere), taking into account the increase of the Doppler shift with distance (Eq. (7)). An example of this spectrum is shown in Fig. 1.

The figure shows that the peak in the $v \cdot f_{v}$ spectrum is indeed the characteristic emitted frequency at the saturation radius (Eq. (29)). The slope of the spectrum above the peak $\beta$ is determined by the power law of the electron distribution through the relation $\beta=-(p-1) / 2$. Below the peak the spectrum shows some curvature (it cannot be exactly fitted with a 
power law) and is a result of the superposition of the contributions from all the radii below the saturation radius.

\section{X-ray flashes}

The origin of X-ray flashes and their connection to GRBs is not clear yet. However, recent analysis (Barraud et al. 2003) has indicated that X-ray flashes actually belong to the same family as GRBs, showing similar spectral characteristics. X-ray flashes appear to be dimmer than GRBs and their peak in the $v \cdot f_{v}$ spectrum is at a photon energy $E_{\mathrm{m}}$ of less than $50 \mathrm{keV}$ (this is actually one way to define X-ray flashes). Barraud et al argue that X-ray flashes may occur at redshifts comparable to those of GRBs since their typical duration does not indicate stronger cosmological time dilation effects (their duration is similar to this of GRBs).

Furthermore, Amati et al. (2002) found indications that $E_{\mathrm{m}}$ correlates with the square root of the equivalent isotropic luminosity released in the GRBs $\left(L \sim X_{\mathrm{ch}, \max }^{1 / 2}\right.$ in our notation). If we assume that this correlation may be extended to the case of X-ray flashes, then a typical X-ray flash, with $E_{\mathrm{m}}$ ten times lower than that of a GRB, would have two orders of magnitude less isotropic luminosity.

So it seems that a more appropriate normalization value of the luminosity per steradian for the typical X-ray flash is $L=$ $10^{49.5}=L_{49.5} \mathrm{erg} / \mathrm{sterad} / \mathrm{s}$. In terms of this model a peak in the spectrum of $\sim 20 \mathrm{keV}$ (which corresponds to $\sim 40 \mathrm{keV}$ in the central engine's frame for $z \sim 1$ ) and using Eq. (29), we find that $\sigma_{0} \simeq 40$. Here we have kept $\epsilon=0.1, \Omega=10^{4} \mathrm{rad} / \mathrm{s}$ and $\xi \sim 10^{-3}$.

The previous argumentation indicates that a wind which is less Poynting-flux dominated (i.e. with higher baryon loading) can give rise to an $\mathrm{X}$-ray flash. For these parameters the saturation radius (Eq. (9)) is $r_{\mathrm{s}} \simeq 2 \times 10^{12} \mathrm{~cm}$, while the photosphere is located at $r_{\mathrm{ph}} \sim 10^{11} \mathrm{~cm}$. For $r_{\mathrm{ph}}<r<r_{\mathrm{s}}$ the radiation emitted has a similar spectrum to that of GRBs (see Fig. 1), but with a peak shifted in the X-ray region (at $\sim 20 \mathrm{keV}$ ). An important fraction of the total energy is, however, dissipated below the photosphere, where scattering plays an important role (see Sect. 7).

\section{The role of synchrotron absorption in the reconnection layers}

The shape of the energy spectrum emitted from the reconnection sites also depends on the synchrotron absorption. The optical depth due to synchrotron absorption is frequency-dependent and at low enough photon energies the medium becomes optically thick and the spectrum hardens. The photon frequency at which the medium makes a transition from the synchrotron thick region to the synchrotron thin region is called the turnover frequency $v_{\mathrm{t}}$. In this section we calculate the turn over frequency as a function in the flow and examine its implications for the emitted spectrum.
An electron of Lorentz factor $\gamma$ in a magnetic field of strength $B^{\prime}$ has a frequency-dependent cross section for synchrotron absorption given by (Ghisellini \& Svensson 1992)

$\sigma_{\mathrm{s}}(v, \gamma)=\frac{3 \pi}{10} \frac{\sigma_{T}}{\alpha_{f}} \frac{B_{\mathrm{cr}}}{B^{\prime}} \frac{x}{\gamma^{5}}\left(K_{4 / 3}^{2}(x / 2)-K_{1 / 3}^{2}(x / 2)\right)$,

where $B_{\text {cr }} \simeq 4.4 \times 10^{13}$ Gauss, $\alpha_{f}$ is the fine structure constant, $x=2 v /\left(3 \gamma^{2} v_{L}\right), v_{L}=e B^{\prime} /(2 \pi m c)$ and $K_{4 / 3}, K_{1 / 3}$ are modified Bessel functions. This expression is valid for $\gamma \gg 1$ and for an isotropic distribution of electrons. Integrating this over a power law distribution of electrons $N(\gamma) \propto \gamma^{-p}$, we find the absorption coefficient

$\alpha_{v}=\int_{\gamma_{\mathrm{ch}}}^{\gamma_{\max }} \sigma_{\mathrm{s}}(\nu, \gamma) N(\gamma) \mathrm{d} \gamma$

where $\gamma_{\mathrm{ch}}$ is given by Eq. (27) and $\gamma_{\max } \gg \gamma_{\mathrm{ch}}$.

The width of the layers (in the comoving frame) is $\delta$ and the turn oner frequency $v_{\mathrm{t}}$ for synchrotron absorption can be estimated solving the equation

$\alpha_{v_{\mathrm{t}}} \cdot \delta=1$.

The thickness of the reconnection layer $\delta$ is related to its typical length $L$ through Eq. (23). This estimate is rather rough; fortunately it turns out that the turn-over frequency depends very weakly on the actual dimensions of the layer.

For the estimate we use as typical parameter values $p \simeq$ $3, \quad \Gamma_{\infty}=1000, \quad \Omega=10^{4} \mathrm{rad} / \mathrm{s}, L=3.2 \times 10^{51} \mathrm{erg} / \mathrm{s} / \mathrm{steradian}$ and $\xi=2 \times 10^{-3}$. Most of the radiation is emitted where most of the energy is dissipated, i.e. near the saturation radius $R_{\mathrm{s}}$ (Eq. (9)). At this distance the turn-over frequency lies in the soft X-rays $\left(v_{\mathrm{t}} \sim 300 \mathrm{eV}\right)$. At smaller radii, the turn-over frequency increases and in the location of the Thomson photosphere it is around $\sim 10 \mathrm{keV}$. Thus synchrotron self-absorption can play a significant role in the shape of the electromagnetic spectrum in the soft X-ray region, making the spectra harder than what is plotted at the low $(E<10 \mathrm{keV})$ energy end of Fig. 1.

\section{Below the photosphere}

Until now, we have explored the radiative properties of the flow in the Thomson-thin region (i.e. $r>r_{\mathrm{ph}}$ ). In this section we briefly discuss the energetics of the quasi-thermal component expected from photons generated below the photosphere. We will call this the photospheric component.

At small radii, or equivalently at large optical depths, radiation and matter are expected to be in thermodynamic equilibrium, sharing the same (comoving) temperature $T$. The thermal energy density, which is dominated by radiation, is fed by the dissipated magnetic energy and suffers from adiabatic losses at the same time. The result is that only a fraction of the energy that was initially injected in thermal form will appear as blackbody radiation when matter and radiation decouple.

The power dissipated as a function of distance is given by Eq. (12). As shown in paper II, half of this energy accelerates the flow, and the other half is injected into the flow as thermal energy. The scaling of this energy rate with radius 
is $\mathrm{d} \dot{E} \propto \mathrm{d} r / r^{2 / 3}$ (Eq. (12)). Suppose now that energy is released at some rate $L_{\text {inj }}$ at a radius $r$ and we want to know how much of it will appear as thermal radiation when matter and radiation decouple at the photosphere. For adiabatic expansion of a radiation-dominated flow, the pressure scales as $\pi \propto \rho^{4 / 3}$ and $\pi \propto T^{4}$. Combining these we have $T \propto \rho^{1 / 3} \propto r^{-7 / 9}$, where in the last step Eq. (13) has been used.

With the scalings $\Gamma \propto r^{1 / 3}$ (Eq. (7)) and $T \propto r^{-7 / 9}$, we have for the luminosity: $L(r) \propto r^{2} \Gamma^{2} T^{4} \propto r^{-4 / 9}$. So, only a fraction $\left(r / r_{\mathrm{ph}}\right)^{4 / 9}$ of the initial thermal energy will still be in thermal form at $r_{\mathrm{ph}}$. So a shell at distance $r, r+\mathrm{d} r$ contributes to the luminosity emitted at $r_{\mathrm{ph}}$

$\mathrm{d} L \propto \frac{\mathrm{d} r}{r^{2 / 3}}\left(\frac{r}{r_{\mathrm{ph}}}\right)^{4 / 9}$.

Integrating the last expression from 0 to $r_{\mathrm{ph}}$ we find for the photospheric luminosity

$L_{\mathrm{ph}}=3 \times 10^{50} L_{52} r_{\mathrm{ph}, 11}^{1 / 3} \frac{(\epsilon \Omega)_{3}^{1 / 3}}{\sigma_{0,2}} \quad \mathrm{erg} / \mathrm{s} / \mathrm{sterad}$.

Where $r_{\text {ph }}$ is given by Eq. (16).

For the typical GRB parameters, one can check that the photospheric component is about one order of magnitude weaker than the total luminosity in the flow $\left(L / L_{\mathrm{ph}} \sim 20\right)$. To find at what frequencies this component is expected to show up, we can use $L_{\mathrm{ph}}$ to calculate the comoving temperature of the flow for a black-body spectrum through the equation $L_{\mathrm{ph}}=16 / 3 \sigma_{S B} r^{2} \Gamma^{2} T^{4}$. Solving for the temperature we have

$T\left(r_{\mathrm{ph}}\right)=\frac{700}{r_{\mathrm{ph}, 11}^{7 / 12}} \frac{L_{52}^{1 / 4}}{(\epsilon \Omega)_{3}^{1 / 12} \sigma_{0,2}^{1 / 2}} \mathrm{eV}$.

Setting again $\sigma_{0}=100, \Omega=10^{4} \mathrm{rad} / \mathrm{s}, L=3.2 \times$ $10^{51} \mathrm{erg} / \mathrm{s} / \mathrm{steradian}$, and $\xi=2 \times 10^{-3}$, we find that the photospheric temperature in the central engine's frame is $T_{\text {ce }}=$ $\Gamma T \simeq 60 \mathrm{keV}$. So, we see that the photospheric emission peaks at similar frequencies to what is typically observed for the non-thermal emission.

At this point it should be emphasized that for a comoving temperature of the order of a few hundred $\mathrm{eV}$ at the photosphere, the approximation of a black-body spectrum emitted at that location may well be poor. This is because at that temperature electron scattering greatly dominates over freefree absorption and cannot in principle be neglected. The same concerns may be raised when the photospheric emission is computed in the "standard" fireball model.

\section{Conclusions}

In this paper we have explored the spectra expected from Poynting-flux dominated outflows and their relevance to GRBs. The field strength in such outflows is much higher than in the usual internal shock models (much above "equipartition" with the radiating fast particles).

We have used the "AC" model, with geometrical configuration similar to the striped wind (Coroniti 1990) in which the magnetic field varies with a typical wavelength of $\lambda \sim 2 \pi c / \Omega$.
The dynamics of this outflow were studied in detail in Papers I, II where it was shown that almost half of the magnetic energy released through magnetic dissipation accelerates the flow to large bulk Lorentz factors. The other half of the magnetic energy accelerates the particles of the flow, mainly above the Thomson photosphere and can serve to power the prompt emission.

An important property of energy dissipation by magnetic reconnection is that the residence time of the accelerated electrons in the reconnection layer is long compared with the synchrotron energy loss time scale. The electrons are thus in approximate balance between heating and cooling, and a sudden acceleration assumption as usually made in (internal) shock acceleration is not valid. In a magnetic model, the break in the Gamma-ray spectrum is therefore unlikely to be associated with the cooling spectrum of suddenly accelerated particles.

We have shown that a GRB-like Gamma-spectrum with a break can be produced by reconnection in a Poynting-flux dominated outflow if the dissipated magnetic energy heats a small fraction $\left(\sim 10^{-4}\right)$ of the electron population. A better understanding of relativistic reconnection will be needed to determine if this number is realistic.

In the magnetic model for GRB emission presented here $\mathrm{X}$-ray flashes are interpreted to belong to the same family as GRBs and are the result of an outflow starting with a smaller magnetization parameter $\sigma$ (higher baryon loading) than needed to produce a GRB. X-ray flashes can also be interpreted within the fireball model with some modification of the parameters.

Finally we have explored the consequences of the strong synchrotron absorption due to the high magnetic fields strengths in a Poynting-flux dominated outflow. We find that it must be important around the electron scattering photosphere, and will result in harder spectra in the soft X-ray range.

Acknowledgements. Giannios acknowledges partial support from the EC Marie Curie Fellowship HPMTCT 2000-00132 and the Program "Heraklitos" of the Ministry of Education of Greece.

\section{References}

Abramowicz, M. A., Novikov, I. D., \& Paczyński, B. 1991, ApJ, 369, 175

Amati, L., Frontera, F., Tavani, M., et al. 2002, A\&A, 390, 81

Band, D., Matteson, J., Ford, L., et al. 1993, ApJ, 413, 281

Barraud, C., Atteia, J. L., Olive, J. F., et al. 2003, A\&A, 400, 1021

Blackman, E. G., \& Field, G. B. 1994a, PhRvL, 71, 3481

Blackman, E. G., \& Field, G. B. 1994b, PhRvL, 73, 3097

Coroniti, F. V. 1990, ApJ, 349, 538

Drenkhahn, G. 2002, A\&A, 387, 714 (Paper I)

Drenkhahn, G., \& Spruit, H. C. 2002, A\&A, 391, 1141 (Paper II)

Frail, D. A., Kulkarni, S. R., Sari, R., et al. 2001, ApJ, 562, L55

Ghisellini, G., \& Svensson, R. 1991, MNRAS, 251, 313

Lyutikov, M., Pariev, V. I., \& Blandford, R. D. 2003, ApJ, 597, 998

Lyutikov, M., \& Blandford, R. D. 2003 [arXiv: astro-ph/0312347]

Lyutikov, M., \& Uzdensky, D. 2003, ApJ, 589, 893

Sikora, M., Begelman, M. C., Coppi, P., \& Proga, D. 2003 [arXiv:astro-ph/0309504]

Spruit, H. C., Daigne, F., \& Drenkhahn, G. 2001, A\&A, 369, 694

Spruit, H. C., \& Drenkhahn, G. 2003 [arXiv: astro-ph/0302468]

Thompson, C. A. 1994, MNRAS, 270, 480 